\title{
A Note on the Maximum Likelihood Estimators for the Mixture of Maxwell Distributions Using Type-I Censored Scheme
}

\author{
Syed Mohsin Ali Kazmi ${ }^{\mathrm{a}, *}$, Muhammad Aslam ${ }^{\mathrm{b}}$ and Sajid $\mathrm{Ali}^{\mathrm{b}}$ \\ ${ }^{a}$ Sustainable Development Policy Institute Islamabad, 44000, Pakistan \\ ${ }^{b}$ Quaid-i-Azam University Islamabad, Pakistan
}

\begin{abstract}
This article focuses on the study of mixture density under Type I censoring scheme by taking Maxwell distribution as a life time model. In this paper we sculpt a heterogeneous population by means of two components mixture of the Maxwell distribution. We derive the maximum likelihood estimators using type-I censored data and also their variances matrix. The problem with ML estimators is discussed. The Maple 13.0 code is also presented in appendix.
\end{abstract}

Keywords: Finite mixture of Maxwell distribution, Censored sampling, Fixed termination time, ML.

\section{INTRODUCTION}

The Maxwell distribution is a probability distribution with application in physics and chemistry. The most frequent application is in the field of statistical mechanics. The temperature of any (massive) physical system is the result of the motions of the molecules and atoms which make up the system. These particles have a range of different velocities, and the velocity of any single particle constantly changes due to collisions with other particles. However, the fraction of a large number of particles within a particular velocity range is nearly constant. Then Maxwell distribution of velocities specifies this fraction, for any velocity range as a function of the temperature of the system.

Censoring is an unavoidable feature of the lifetime applications and in form of missing data problem. An account of censoring can be seen in Leemis [1], Dietz, Gail and Krickeberbg [2], Klein and Moeschberger [3], Kalbfleisch and Prentice [4] and Smith [5] which are valuable contribution on survival analysis technique for censored and truncated data. Jiang and Kececioglu [6] deals with maximum likelihood estimates using censored data for mixed Weibull distribution while Wang and $\mathrm{Li}$ [7] considers the estimators for survival function when censoring times are known. Censoring is divided into three types. i.e. left, right, and interval censoring. Right censoring may be of type-I, type-II or random right censoring. The type-I is further divided into ordinary type-I, progressive type-I and generalized type-I censoring while the type-II sampling is categorized as ordinary type-II and progressive type-II censoring. An observation on lifetime of an object is said to be censored one if the exact life length of that object is unknown. Censoring is called right (left) if the unknown life length lies on the right (left) of the end (start) of study. The random right censoring is said to be employed if lifetime of an object is greater than an independent random umber. In type-I (type-II) right censor-

*Address correspondence to this author at the Sustainable Development Policy Institute Islamabad, 44000, Pakistan; Tel: +923346545319;

E-mail: mohsinstats@yahoo.com ing the life-test termination time (the number of dead objects) is pre-specified. In ordinary type-I censoring right censoring the life-test termination time is the same for all objects. The lifetime of an object is called interval censored if it is known to fall between a known time-interval. In our study, an ordinary type-I, right censoring is considered with a fixed-test termination time.

Tyagi and Bhattacharya (a) [8], Tyagi and Bhattacharya (b) [9] considered Maxwell distribution as a lifetime model for the first time. They obtained Bayes estimates and minimum variance unbiased estimators of the parameter and reliability function for the Maxwell distribution. Chaturvedi and Rani [10] generalized Maxwell distribution and they obtained Classical and Bayesian estimators for generalized distribution. Bekker and Roux [11] studied Empirical Bayes estimation for Maxwell distribution. These studies give mathematical handling to Maxwell distribution but ignore the application aspect of the Maxwell distribution.

A finite mixture of some suitable probability distribution is recommended to study a population that is supposed to comprise a number of subpopulations mixed in unknown proportion. A population of lifetimes of certain electrical elements may be divided into a number of subpopulations depending upon the possible case. Mixture model have been used in physical, chemical, social science, biological and other fields. For example Sinha [12] considered the Bayesian counterpart of the maximum likelihood estimates of the Mendenhall and Hader [13] mixture.

In this paper, random observations taken from this population are supposed to be characterized by one of the two distinct unknown members of a Maxwell distribution. So the two component mixture of the Maxwell distribution is recommended to model this population. Right censoring is considered and the observations greater than the fixed cut off censor value, $T$ are taken as censored ones.

The Maxwell mixture model is defined in Section 2 and its likelihood is developed in Section 3. Section 4 contains the variance equations of ML. Some concluding remarks are given in last Section 5. 


\section{THE MAXWELL MIXTURE MODEL}

A finite mixture density function with two component densities of specified parametric form with two unknown mixing parameters, $\theta_{i}, i=1,2$ and with two mixing weights $(p, 1-p)$ is defined as follows

$$
f(x)=p f_{1}(x)+(1-p) f_{2}(x), 0<p<1
$$

The following Maxwell distribution is assumed for ith components of the mixture, $i=1$ or 2

$$
f_{i}(x)=\frac{4}{\sqrt{\pi}} \frac{1}{\theta_{i}^{\frac{3}{2}}} x^{2} e^{-\frac{x^{2}}{\theta_{i}}}, \theta_{i}>0,0 \leq x \leq \infty, i=1,2
$$

so the mixture model (1) takes the following form

$$
f(x)=p\left(\frac{4}{\sqrt{\pi}} \frac{1}{\theta_{1}^{\frac{3}{2}}} x^{2} e^{-\frac{x^{2}}{\theta_{1}}}\right)+q\left(\frac{4}{\sqrt{\pi}} \frac{1}{\theta_{2}^{\frac{3}{2}}} x^{2} e^{-\frac{x^{2}}{\theta_{2}}}\right)
$$

where $q=1-p, 0<p<1$.

The cumulative distribution function (cdf) of the Maxwell distribution is give by:

$$
F(x)=2 \operatorname{erf}\left(\frac{x^{2}}{\sqrt{\theta}}\right)-\frac{2}{\sqrt{\pi}} \frac{x}{\sqrt{\theta}} e^{-\frac{x^{2}}{\theta}},
$$

where $\operatorname{erf}(x)=\frac{2}{\sqrt{\pi}} \int_{0}^{x} e^{-u^{2}} d u$ is the well-known error function.

Krishna and Malik [14] use the following form of distribution function:

$$
F(x)=1-\frac{1}{\theta^{\frac{3}{2}}} \frac{4}{\sqrt{\pi}} J(x, 2, \theta),
$$

where

$$
J(x, k, \theta)=\int_{0}^{x} e^{-\frac{u^{2}}{\theta}} u^{k} d u .
$$

Bekker and Roux [1] introduce another form of distribution function

$$
F(x)=\frac{2}{\sqrt{\pi}} \Gamma\left(\frac{x^{2}}{\theta}, \frac{3}{2}\right)
$$

where $\Gamma(x, a)=\int_{0}^{x} e^{-u} u^{a-1} d u$, is the incomplete gamma function.

So by using mixing weights the cumulative distribution function become

$$
F(x)=p F_{1}(x)+(1-p) F_{2}(x)
$$

Where $F_{i}(x)=\frac{2}{\sqrt{\pi}} \Gamma\left(\frac{x^{2}}{\theta_{i}}, \frac{3}{2}\right)$, while $i=1,2$

$F(x)=p\left\{\frac{2}{\sqrt{\pi}} \Gamma\left(\frac{x^{2}}{\theta_{1}}, \frac{3}{2}\right)\right\}+(1-p)\left\{\frac{2}{\sqrt{\pi}} \Gamma\left(\frac{x^{2}}{\theta_{2}}, \frac{3}{2}\right)\right\}$,

$\theta_{1}, \theta_{2}>0$

\section{LIKELIHOOD FUNCTION}

Suppose $\mathrm{n}$ units from the above mixture model are used to life testing experiment. . As discussed in section 1 right censoring is carried out using either a fixed censoring time $t$. All observations which are greater than $T$ are stated as censored ones. Different fixed censoring times $t$ are chosen to evaluate the impact of censoring rate on the estimates. By using this censoring scheme let the test be conducted and at fixed censoring time $t$, it is observed that out of $n$ units, test is terminated as $r$ th failure occurs and the remaining $n-r$ units are still working. Mendenhall and Hader [13] enlighten that in many real life situations only the failed objects can easily be identified as member of either subpopulation 1 or subpopulation 2. So, depending upon the cause of failure it may be observed that $r_{1}$ and $r_{2}$ are identified as members of the first and second subpopulation respectively. It is apparent that $r=r_{1}+r_{2}$ and remaining $n-r$ objects provide no information about the subpopulation to which they belong. We define, $x_{i j}$ as the failure time of the $j$ th observation in the ith subpopulation, where $i=1$ or 2 ; and $j=1,2,3, \ldots \ldots . . r_{i} ; \quad 0<x_{1 j}, x_{2 j} \leq \infty$.So the likelihood function for the given condition is:

$$
L\left(\theta_{1}, \theta_{2}, p \mid \mathrm{x}\right) \propto\left\{\prod_{j=1}^{r_{i}} p f_{1}\left(x_{1 j}\right)\right\}\left\{\prod_{j=1}^{r_{2}} q f_{2}\left(x_{2 j}\right)\right\}\left\{(1-F(t))^{n-r}\right\}
$$

where $\mathrm{x}=\left(x_{11}, x_{12} \ldots \ldots, x_{1 r_{1}}, x_{21}, x_{22}, \ldots . ., x_{2 r_{2}}\right)$ is the observed failure times for the non-censored observations and likelihood function provided as

$L\left(\theta_{1}, \theta_{2}, p \mid \mathbf{x}\right) \propto\left\{\prod_{j=1}^{r} p\left(\frac{4}{\sqrt{\pi}} \frac{1}{\theta_{1}^{\frac{3}{2}}} x_{1 j}^{2} e^{\frac{x_{i j}^{2}}{\theta_{1}}}\right)\right]\left\{\prod_{j=1}^{r_{2}} q\left(\frac{4}{\sqrt{\pi}} \frac{1}{\theta_{2}^{\frac{3}{2}}} x_{2 j}^{2} e^{\frac{-r_{2}}{\theta_{2}}}\right)\right]\left[\{1-F(t)\}^{n-r}\right]$

where $F(t)=\frac{2 p}{\sqrt{\pi}} \Gamma\left(\frac{t_{1}^{2}}{\theta_{1}}, \frac{3}{2}\right)+\frac{2 q}{\sqrt{\pi}} \Gamma\left(\frac{t_{2}^{2}}{\theta_{2}}, \frac{3}{2}\right)$,

and $1-F(t)=1-\frac{2}{\sqrt{\pi}}\left\{p \Gamma\left(\frac{t_{1}^{2}}{\theta_{1}}, \frac{3}{2}\right)+q \Gamma\left(\frac{t_{2}^{2}}{\theta_{2}}, \frac{3}{2}\right)\right\}$,

where $1-F(t)$ is known as survival function. According to the Type I Censoring scheme, time is fixed so $t_{1}=t_{2}=t$

After some manipulation the likelihood function (6.8) takes the following form: 


$$
\begin{aligned}
& L\left(\theta_{1}, \theta_{2}, p \mid \mathrm{x}\right) \propto \sum_{k=0}^{n-r} \sum_{m=0}^{k}(-1)^{k}\left(\begin{array}{c}
n-r \\
k
\end{array}\right)\left(\begin{array}{c}
k \\
m
\end{array}\right) p^{r_{1}}\left(\frac{4}{\sqrt{\pi}}\right)^{r_{1}} \frac{1}{\theta_{1}^{\frac{3 r_{1}}{2}}} \prod_{j=1}^{r_{1}} x_{1 j}{ }^{2} e^{\sum_{i=1}^{n} x_{1 i}{ }^{2}} \times \\
& q^{r_{2}}\left(\frac{4}{\sqrt{\pi}}\right)^{r_{2}} \frac{1}{\theta_{2}{ }^{\frac{3 r_{2}}{2}}} \prod_{j=1}^{r_{2}} x_{2 j}{ }^{2} e^{-\sum_{j=1}^{\eta_{j}} x_{2 j}{ }^{2}}\left\{\frac{p}{0.5 \sqrt{\pi}} \Gamma\left(\frac{t^{2}}{\theta_{1}}, \frac{3}{2}\right)\right\}^{k-m}\left\{\frac{q}{0.5 \sqrt{\pi}} \Gamma\left(\frac{t^{2}}{\theta_{2}}, \frac{3}{2}\right)\right\}^{m} \\
& L\left(\theta_{1}, \theta_{2}, p \mid \mathrm{x}\right) \propto \sum_{k=0}^{n-r} \sum_{m=0}^{k}(-1)^{k}\left(\begin{array}{c}
n-r \\
k
\end{array}\right)\left(\begin{array}{l}
k \\
m
\end{array}\right) p^{r_{1}+k-m} q^{r_{2}+m}\left(\frac{4}{\sqrt{\pi}}\right)^{r_{1}}\left(\frac{4}{\sqrt{\pi}}\right)^{r_{2}} \prod_{j=1}^{r_{1}} x_{1 j}{ }^{2} \prod_{j=1}^{r_{2}} x_{2 j}{ }^{2} \times \\
& \frac{1}{\theta_{1}^{\frac{3 r_{1}}{2}}} e^{-\frac{\sum_{j=1}^{r_{1 j}} x_{1 j}{ }^{2}}{\theta_{1}}} \frac{1}{\theta_{2}^{\frac{3 r_{2}}{2}}} e^{-\frac{\sum_{j=1}^{r_{2} x_{2 j}{ }^{2}}}{\theta_{2}}} D_{1}^{k-m} D_{2}^{m} \\
& \ln \left(L\left(\theta_{1}, \theta_{2}, p \mid \mathrm{x}\right)\right) \propto \ln \sum_{k=0}^{n-r} \sum_{m=0}^{k}(-1)^{k}\left(\begin{array}{c}
n-r \\
k
\end{array}\right)\left(\begin{array}{c}
k \\
m
\end{array}\right) p^{r_{1}+k-m} q^{r_{2}+m}\left(\frac{4}{\sqrt{\pi}}\right)^{r_{1}}\left(\frac{4}{\sqrt{\pi}}\right)^{r_{2}} \prod_{j=1}^{r_{1}} x_{1 j}{ }^{2} \prod_{j=1}^{r_{2}} x_{2 j}{ }^{2} \times{ }^{\prime} \\
& \frac{1}{\theta_{1}^{\frac{3 r_{1}}{2}}} e^{-\frac{\sum_{j=1}^{r_{1}} x_{1 j}{ }^{2}}{\theta_{1}}} \frac{1}{\theta_{2}{ }^{\frac{3 r_{2}}{2}}} e^{-\frac{\sum_{j=1}^{r_{2}} x_{2 j}{ }^{2}}{\theta_{2}}} D_{1}^{k-m} D_{2}^{m} \\
& \frac{\partial l}{\partial \theta_{1}}=-\frac{3 r_{1}}{2 \theta_{1}}+\frac{\sum_{i=1}^{r_{1}} x_{1 j}^{2}}{\theta_{1}^{2}}+\frac{(k-m)}{Z_{1}}\left(\frac{\partial}{\partial \theta_{1}} Z_{1}\right)=0
\end{aligned}
$$

where

$$
D_{i}=\frac{1}{0.5 \sqrt{\pi}} \Gamma\left(\frac{t^{2}}{\theta_{i}}, \frac{3}{2}\right) .
$$

Here problem arise during finding solution of these equations because parameters appear in integration limit. So we cannot get simplified solution.

\section{MAXIMUM LIKELIHOOD ESTIMATES}

The sampling scheme includes sample of size $\mathrm{n}$ units from the mixture model described above under the ordinary type-I censoring. Let a minor inspection of the dead objects shows that $r t h$ failure occurs and remaining $n-r$ objects still alive and hence cannot be labeled because of censoring. The maximum likelihood estimates of parameters $\theta_{1}, \theta_{2}$ and $p$, using (9) are attained by solving nonlinear equations (10) to (12). These equation are obtained by differentiating the natural $\log$ of likelihood (8) with respect to $\theta_{1}, \theta_{2}$ and $p$ respectively and equating first order partial derivative to zero.

$$
\frac{\partial l}{\partial \theta_{2}}=-\frac{3 r_{2}}{2 \theta_{2}}+\frac{\sum_{i=1}^{r_{2}} x_{2 j}^{2}}{\theta_{2}^{2}}+\frac{(k-m)}{Z_{2}}\left(\frac{\partial}{\partial \theta_{2}} Z_{2}\right)=0
$$

Since in both cases the differentiation is with respect to $\theta_{1}$ and $\theta_{2}$ while here both $\theta_{1}, \theta_{2}$ are not in the limits of the gamma function therefore the fundamental theorem of calculus is not applicable and hence not simple results are possible.

Similarly

$$
\frac{\partial l}{\partial p}=\frac{r_{1}+k-m}{p}-\frac{r_{2}+m}{(1-p)}-\frac{2(n-r)\left(Z_{1}-Z_{2}\right)}{\sqrt{\pi}\left(1-\frac{2}{\sqrt{\pi}}\left(p Z_{1}+(1-p) Z_{2}\right)\right)}=0
$$

where

$$
Z_{i}=\Gamma\left(\frac{T^{2}}{\theta_{i}}, \frac{3}{2}\right)
$$

\section{VARIANCE OF THE MAXIMUM LIKELIHOOD ESTIMATES}

Now Variances of the maximum likelihood estimates of $\theta_{1}, \theta_{2}$ and $p$ are derived by Information matrix using the expectation of the negative Hessian. The variances of ML estimates are on the main diagonal of the inverted Information matrix given below 


$$
\begin{aligned}
& I\left(\theta_{1} ; \theta_{2} ; p\right)=-E\left[\begin{array}{ccc}
\frac{\partial^{2} l}{\partial \theta_{1}^{2}} & \frac{\partial^{2} l}{\partial \theta_{1} \partial \theta_{2}} & \frac{\partial^{2} l}{\partial \theta_{1} \partial p} \\
\frac{\partial^{2} l}{\partial \theta_{2} \partial \theta_{1}} & \frac{\partial^{2} l}{\partial \theta_{2}^{2}} & \frac{\partial^{2} l}{\partial \theta_{2} \partial p} \\
\frac{\partial^{2} l}{\partial p \partial \theta_{1}} & \frac{\partial^{2} l}{\partial p \partial \theta_{2}} & \frac{\partial^{2} l}{\partial p^{2}}
\end{array}\right] . \\
& \frac{\partial^{2} l}{\partial \theta_{1}^{2}}=\frac{3 r_{1}}{2 \theta_{1}^{2}}-\frac{2 \sum_{j=1}^{r_{1}} x_{1 j}^{2}}{\theta_{1}^{3}}+\frac{Z_{1} \frac{\partial^{2}}{\partial \theta_{1}^{2}}\left(Z_{1}\right)-\left(\frac{\partial}{\partial \theta_{1}} Z_{1}\right)^{2}}{\left(Z_{1}\right)^{2}} \\
& \frac{\partial^{2} l}{\partial \theta_{2}^{2}}=\frac{3 r_{2}}{2 \theta_{2}^{2}}-\frac{2 \sum_{j=1}^{r_{2}} x_{2 j}^{2}}{\theta_{2}^{3}}+\frac{Z_{2} \frac{\partial^{2}}{\partial \theta_{2}^{2}}\left(Z_{2}\right)-\left(\frac{\partial}{\partial \theta_{2}} Z_{2}\right)^{2}}{\left(Z_{2}\right)^{2}} \\
& \frac{\partial^{2} l}{\partial p^{2}}=-\frac{r_{1}+k-m}{p^{2}}+\frac{r_{2}+m}{(1-p)^{2}}-\frac{2(n-r)\left(Z_{1}-Z_{2}\right)^{2}}{\sqrt{\pi}\left(1-\frac{2}{\sqrt{\pi}}\left(p Z_{1}+(1-p) Z_{2}\right)\right)^{2}}
\end{aligned}
$$

The computation of the above elements of Information matrix can be conducted using Mathematica software after replacing the parameters by their estimates obtained by equations (10) to (12).

\section{CONCLUSION}

Here we have derived the maximum likelihood estimators and their respective variances matrix for the mixture of Maxwell model using Type-I censored data. A Maple 13.0 code is also presented in appendix for the maximum likelihood equation solution. Since the maximum likelihood equations involve the parameters in limit of integral, so we cannot get simplified solution of these parameters values and their variances.

\section{ACKNOWLEDGEMENT}

Dr. Hare Krishna (Chaudhary Charan Singh University, Meerut, U.P. India), Dr. Abid Suleri (Sustainable Development Policy Institute, Islamabad. Pakistan) and referee's of Open Statistics and Probability Journal, who deserves special thanks for his encouragement and providing us useful comments in order to presents this research work in a beautiful manner.

\section{APPENDIX}

The following Maple Code can be used for obtaining MLE and their variance matrix component.

$$
\begin{aligned}
\ln L:=c+r_{1} \ln (p)-1.5 r_{1} \ln \left(\theta_{1}\right)-\frac{\sum_{j=1}^{r_{1}} x_{1 j}^{2}}{\theta_{1}}+r_{2} \ln (1-p) \\
-1.5 r_{2} \ln \left(\theta_{2}\right)-\frac{\sum_{j=1}^{r_{2}} x_{2 j}^{2}}{\theta_{2}}+(n-r) \ln \left(1-\frac{2}{\sqrt{\pi}}\left\{p \Gamma\left(\frac{T^{2}}{\theta_{1}}, \frac{3}{2}\right)\right.\right. \\
\left.\left.+(1-p) \Gamma\left(\frac{T^{2}}{\theta_{2}}, \frac{3}{2}\right)\right\}\right)
\end{aligned}
$$

$$
\begin{aligned}
& l p:=(\operatorname{simplify}(\operatorname{diff}(\ln L,(p))=0,(p))) \\
& \text { lth1 := } \left.\operatorname{simplify}\left(\operatorname{diff}\left(\ln L,\left(\theta_{1}\right)\right)=0,\left(\theta_{1}\right)\right)\right) \\
& \text { convert }\left(\operatorname{MeijerG}\left([[],[1,1]],\left[\left[0,0, \frac{T^{2}}{\theta_{1}}\right],[]\right], \frac{3}{2}\right)\right. \text {, } \\
& \text { 'StandardFunctions ') } \\
& \text { lth2 := }\left(\operatorname{simplify}\left(\operatorname{diff}\left(\ln L,\left(\theta_{2}\right)\right)=0,\left(\theta_{2}\right)\right)\right) \\
& \text { convert }\left(\operatorname{MeijerG}\left([[],[1,1]],\left[\left[0,0, \frac{T^{2}}{\theta_{2}}\right],[]\right], \frac{3}{2}\right)\right. \text {, } \\
& \text { 'StandardFunctions ' } \\
& \text { lthls }:=\left(\operatorname{simplify}\left(\operatorname{diff}\left(\ln L,\left(\theta_{1} \$ 2\right)\right),\left(\theta_{1}\right)\right)\right) \\
& \text { lth } 2 s:=\left(\operatorname{simplify}\left(\operatorname{diff}\left(\ln L,\left(\theta_{2} \$ 2\right)\right),\left(\theta_{2}\right)\right)\right) \\
& \text { lps }:=(\operatorname{simplify}(\operatorname{diff}(\ln L,(p \$ 2)),(p))) \\
& \left(\operatorname{simplify}\left(\operatorname{diff}\left(\ln L,\left(\theta_{1}, \theta_{2}\right)\right),\left(\theta_{1}, \theta_{2}\right)\right)\right) \\
& \text { lpth1 }:=\left(\operatorname{simplify}\left(\operatorname{diff}\left(\ln L,\left(\theta_{1}, p\right)\right),\left(\theta_{1}, p\right)\right)\right) \\
& \text { lth12:= }\left(\operatorname{simplify}\left(\operatorname{diff}\left(\ln L,\left(\theta_{1}, \theta_{2}\right)\right),\left(\theta_{1}, \theta_{2}\right)\right)\right) \\
& \text { lpth2 : }=\left(\operatorname{simplify}\left(\operatorname{diff}\left(\ln L,\left(p, \theta_{2}\right)\right),\left(p, \theta_{2}\right)\right)\right) \\
& \operatorname{diff}\left(\ln L,\left(\theta_{1}, \theta_{2}\right)\right),\left(\operatorname{simplify}\left(\operatorname{diff}\left(\Gamma\left(\frac{T^{2}}{\theta_{1}}, \frac{3}{2}\right),\left(\theta_{1}\right)\right),\left(\theta_{1}\right)\right)\right) \\
& A:=\operatorname{Matrix}([\text { lth } 1 \text { s, lth12, lth21 },[\text { lth21, lth2s, lth12 }\rceil,[\text { lpth1, lpth2lps }]) \\
& B:=\operatorname{Inverse}(A)
\end{aligned}
$$

\section{REFERENCES}

[1] L.M. Leemis, Reliability - Probabilistic Models and Statistical Methods. Prentice Hall, Inc., Englewood Cliffs: New Jersey, 1995.

[2] K. Dietz., M. Gail, K. Krickeberg., and B.Singer, "Statistics in the health sciences." Springer-Verlag New York, Inc: New York, 1995.

[3] J. Klein, and M. Moeschberger, "Survival Analysis Techniques for Censored and Truncated Data. Springer-Verlag Inc: New York, 1997.

[4] J.D. Kalbfleisch, and R.L. Prentice, "The Statistical Analysis of Failure Time Data” John Wiley \& Sons, Inc: New York, 2002.

[5] P.J. Smith, "Analysis of Failure and Survival Data." Chapman \& Hall/CRC: New York, 2002

[6] S.Jiang, and D. Kececioglu, "Maximum Likelihood estimates, from censored data, for mixed-Weibull distributions", IEEE Trans. Reliab., vol. 41(2): pp. 241-247, 1992.

[7] J. Wang, and $\mathrm{Y}$. $\mathrm{Li}$, "Estimators for survival function when censoring time are known" Commun Stat Theory Graph Stat, 10(3): pp. 440-454, 2005

[8] R.K. Tyagi, and S.K. Bhattacharya, "Bayes estimation of the Maxwell's velocity distribution function", Stistica., Vol. 29. no 4, pp. 563-7, 1989

[9] R.K. Tyagi, and S.K. Bhattacharya, "A note on the MVU estimation of reliability for the Maxwell failure distribution", Estadistica., Vol.41 no. 137, 1989b.

[10] Chaturvedi, and U, Rani, "Classical and Bayesian reliability estimation of the generalized Maxwell failure distribution", J. Stat Res., Vol. 32, pp. 113-120, 1998.

Bekker, and J.J. Roux, "Reliability characteristics of the Maxwell distribution: a Bayes estimation study", Comm. Stat, (Theory \& Meth), Vol. 34 no. 11, pp. 2169-78, 2005.

[12] S.K. Sinha, Bayesian Estimation." $1^{\text {st }}$ ed. New Age International (P) limited ,Publisher, New Delhi, 1998 
[13] W. Mendenhall, and R.J. Hader, "Estimation of Parameters of mixed exponentially distributed failure time distributions from censored life test data". Biometrika., vol.45, pp. 504-520, 1958.
[14] Krishna, H and M. Malik, "Reliability estimation in Maxwell distribution with Type-II censored data", Int. J. Quality. Reliab Manag., Vol.26, no.2, pp. 184-195, 2009.

Received: September 02, 2011

Revised: October 12, 2011

Accepted: October 18, 2011

(C) Kazmi; Licensee Bentham Open.

This is an open access article licensed under the terms of the Creative Commons Attribution Non-Commercial License (http://creativecommons.org/licenses/by-nc/3.0/) which permits unrestricted, non-commercial use, distribution and reproduction in any medium, provided the work is properly cited. 